\title{
A Limit EQUATION ASSOCIATED TO THE SOLVABILITY OF THE VACUUM EINSTEIN CONSTRAINT EQUATIONS USING THE CONFORMAL METHOD
}

\author{
MATTIAS DAHL, ROMAIN GICQUAUD, AND EMMANUEL HUMBERT
}

\begin{abstract}
Let $(M, g)$ be a compact Riemannian manifold on which a tracefree and divergence-free $\sigma \in W^{1, p}$ and a positive function $\tau \in W^{1, p}, p>n$, are fixed. In this paper, we study the vacuum Einstein constraint equations using the well known conformal method with data $\sigma$ and $\tau$. We show that if no solution exists then there is a non-trivial solution of another non-linear limit equation on 1-forms. This last equation can be shown to be without solutions no solution in many situations. As a corollary, we get existence of solutions of the vacuum Einstein constraint equation under explicit assumptions which in particular hold on a dense set of metrics $g$ for the $C^{0}$-topology.
\end{abstract}

\section{Contents}

1. Introduction

1.1. Background

1.2. Statement of results

2. Proof of Theorem 1.1

2.1. The subcritical system

2.2. Convergence of subcritical solutions

3. The limit equation: existence and non-existence results

3.1. Non-existence results

3.2. Existence result for the limit equation 17

4. Proof of Theorem 1.7 19

Appendix A. Positivity of $C_{g}$

References

\section{INTRODUCTION}

1.1. Background. The Cauchy problem in general relativity asks for a space-time development of a given initial data set $(M, g, K)$ consisting of an $n$-dimensional manifold $M$ equipped with a Riemannian metric $g$ and a symmetric $(0,2)$-tensor $K$. Such a development is a manifold $\mathcal{M}:=M \times \mathbb{R}$ equipped with a globally

Date: January 28, 2011.

2010 Mathematics Subject Classification. 53C21 (Primary), 35Q75, 53C80, 83C05 (Secondary).

Key words and phrases. Einstein constraint equations, non-constant mean curvature, conformal method. 
hyperbolic Lorentzian metric $G$ satisfying Einstein's equation

$$
\operatorname{Ric}^{G}-\frac{1}{2} R^{G} G=8 \pi T .
$$

Here $\operatorname{Ric}^{G}$ and $R^{G}$ are the Ricci and scalar curvatures of the metric $G$ and $T$ is the energy-momentum tensor modeling the matter. For $(\mathcal{M}, G)$ to be a development it is required that $(M, g)$ embeds isometrically on the slice $M \times\{0\}$ with second fundamental form $K$.

The initial data $(M, g, K)$ cannot be freely specified but must itself satisfy the so-called the Einstein constraint equations. In this paper, we are interested in the Vacuum Einstein constraint equations

$$
\begin{aligned}
R-|K|^{2}+(\operatorname{tr} K)^{2} & =0, \\
\operatorname{div} K-d \operatorname{tr} K & =0 .
\end{aligned}
$$

corresponding to the case where the energy-momentum tensor $T$ vanishes everywhere. Y. Choquet-Bruhat and R. Geroch [6] proved that such initial data give rise to a unique development $(\mathcal{M}, G)$ as described above.

The most efficient method to find initial data satisfying the vacuum constraint equations is the conformal method developed by A. Lichnerowicz [16] and Y. Choquet-Bruhat and J. W. York [7]. For this approach we let $(M, g)$ be a Riemannian manifold, $\tau$ a smooth function and $\sigma$ be a trace-free and divergence-free symmetric $(0,2)$-tensor on $M$. We consider the following system of equations for $\varphi$ and $W$,

$$
\begin{aligned}
\frac{4(n-1)}{n-2} \Delta \varphi+R \varphi & =-\frac{n-1}{n} \tau^{2} \varphi^{N-1}+|\sigma+L W|^{2} \varphi^{-N-1}, \\
-\frac{1}{2} L^{*} L W & =\frac{n-1}{n} \varphi^{N} d \tau,
\end{aligned}
$$

where $\varphi$ is a positive smooth and where $W$ is a smooth one-form. In this system the first equation is called the Lichnerowicz equation, and the second equation is called the vector equation. These coupled equations are together called the conformal constraint equations. We have set $N:=\frac{2 n}{n-2}$. Further, $\Delta$ is the non-negative Laplacian acting on functions, $R$ the scalar curvature, and $L$ the conformal Killing operator, all defined using the metric $g$. Except when the notation indicates otherwise all metric-dependent objects on $M$ are assumed to be defined using the metric $g$. The conformal Killing operator is defined on a 1-form $V$ by taking the symmetric, trace-free part of $\nabla V$. The vector fields corresponding to 1-forms in the kernel of $L$ are precisely the conformal Killing vector fields, to assume that $(M, g)$ has no conformal Killing vector fields is thus equivalent to assume that $\operatorname{ker} L=0$.

If $(\varphi, W)$ are solutions of the conformal constraint equations (2a)-(2b) then

$$
\left(M, \varphi^{N-2} g, \frac{\tau}{n} \varphi^{N-2} g+\varphi^{-2}(\sigma+L W)\right)
$$

is an initial data set satisfying the vacuum Einstein's constraint equations (1a)(1b), see for example [3] or [5, Chapter VII]. Note that the function $\tau$ is then the mean curvature of the embedding of $(M, g)$ into $(\mathcal{M}, G)$.

Let us now describe the results on existence of solutions to the system (2a)-(2b) which are known until now. We restrict to the case of closed manifolds $M$. The case of constant functions $\tau$ is completely understood, see [13], while the case of non-constant $\tau$ in its full generality is still open. All the known existence results 
require that $d \tau / \tau$ is small or that $\sigma$ is small. The reader can refer for instance to [14, 1], or [19. In the presence of matter, then again some smallness of the data is needed, see [11. Some results are known for initial data of lower regularity, see [12, 18].

The main result of this paper, Theorem 1.1, is that if the conformal constraint equations (2a)-(2b) have no solution, then there exists a non-trivial 1-form $W$ solving the equation

$$
-\frac{1}{2} L^{*} L W=\alpha_{0} \sqrt{\frac{n-1}{n}}|L W| \frac{d \tau}{\tau}
$$

for some $\alpha_{0} \in(0,1]$. This result has advantages compared to previous results:

- In the case that Equation (3) has no solution for any $\alpha_{0} \in(0,1]$, then we obtain that there exists a solution of the conformal constraint equations (2a) - (2b). We also obtain the compactness of the set of solutions.

- In the case that Equation (3) has no solution, $g, \tau$ are in the interior of the set of data for which (2a)-2b) have solutions for any $\sigma$, see Proposition 1.2 .

- If $d \tau / \tau$ is small enough, then Equation (3) has no solution. The point here is that the smallness condition is explicit, see Corollaries 1.3 and 1.4

- We do not need to assume that the initial data $g, \tau, \sigma$ are smooth.

- This is maybe the main point: the assumptions required in Corollary 1.3 hold for a dense set of metrics with respect to the $C^{0}$-topology. In other words, let $\tau$ be fixed with constant sign. Then, there exists a dense set of metrics with respect to the $C^{0}$-topology such that for all $\sigma$, we get solutions of Equations (2a)-(2b), see Corollary 1.5. To our knowledge, this is the first result showing that the set of solutions of the conformal constraint equations is large. Furthermore, it was not known that for any such $\tau$, we can find even one metric giving rise to solutions of the conformal constraint equations.

- Theorem 1.1 lets us expect some further developments by finding other situations where Equation (3) has no solution.

The method we use to prove our main result is to decrease the exponent $N$ of $\varphi$ in the right hand side of the vector equation (2b) to $N-\epsilon$, this idea was introduced to us by J. Isenberg. The new equations obtained in this way we call "subcritical" (even though the situation here is not related to criticality of Sobolev embedding as for the Yamabe problem). At a certain point of the proof we can find a supersolution for the subcritical equations regardless of the size of the coefficients in the equations, this makes it possible to solve the subcritical equations for all $\epsilon>0$. In (12) below we define an "energy" for solutions to the subcritical equations. It turns out that the behavior of solutions as $\epsilon \rightarrow 0$ is controlled by this energy, if it is bounded we get convergence to a solution of the conformal constraint equations, otherwise certain rescaled solutions converge to a solution to the limit equation.

There are only few results concerning non-existence of solutions of the conformal constraint equations. In [15, Theorem 2] it is shown that there are no solutions if $\sigma \equiv 0$ and $d \tau / \tau$ is small enough. In Theorem 1.7 we strengthen this result by giving a more explicit bound on $d \tau / \tau$.

1.2. Statement of results. Let $M$ be a compact manifold of dimension $n$, our goal is to find solutions to the vacuum Einstein constraint equations using the 
conformal Method. The given data on $M$ consists of

- a Riemannian metric $g \in W^{2, p}$,

- a function $\tau \in W^{1, p}$,

- a symmetric, trace- and divergence-free $(0,2)$-tensor $\sigma \in W^{1, p}$,

with $p>n$, and one is required to find

- a positive function $\varphi \in W^{2, p}$,

- a 1-form $W \in W^{2, p}$,

which satisfy the conformal constraint equations (2a) $-(2 \mathrm{~b})$.

We use standard notation for function spaces, such as $L^{p}, C^{k}$, and Sobolev spaces $W^{k, p}$. It will be clear from the context if the notation refers to a space of functions on $M$, or a space of sections of some bundle over $M$. For spaces of functions the subscript + is used to indicate the subspace of positive functions.

In our existence result we will assume that

- $\tau$ vanishes nowhere,

- $(M, g)$ has no conformal Killing vector fields,

- $\sigma \not \equiv 0$ if $Y(g) \geq 0$.

Here $Y(g)$ is the Yamabe constant of the conformal class of $g$, that is

$$
Y(g):=\inf \frac{\int_{M} R^{\widetilde{g}} d v^{\widetilde{g}}}{\operatorname{vol}^{\widetilde{g}}(M)^{\frac{n-2}{n}}},
$$

where the infimum is taken over all $\widetilde{g} \in W^{2, p}$ conformal to $g$. Our main result is the following Theorem.

Theorem 1.1. Let data be given on $M$ as specified in (41) and assume that (5) holds. Then, at least one of following assertions is true.

- The system of equations (2a)-(2b) admits a solution $(\varphi, W)$ with $\varphi>0$. Furthermore, the set of solutions $(\varphi, W) \in W_{+}^{2, p} \times W^{2, p}$ is compact.

- There exists a non-trivial solution $W \in W^{2, p}$ of the equation

$$
-\frac{1}{2} L^{*} L W=\alpha_{0} \sqrt{\frac{n-1}{n}}|L W| \frac{d \tau}{\tau}
$$

for some $\alpha_{0} \in(0,1]$.

We will call Equation (6) the limit equation since it appears as the equation satisfied by a limit of rescaled solutions. As an immediate consequence we conclude that if Equation (6) has no solution for any $\alpha_{0} \in(0,1]$, then the conformal constraint equations (2a)-(2b) admit a solution $(\varphi, W)$ with $\varphi>0$. Note that a solution to the limit equation with $\alpha_{0}=0$ would be a conformal Killing field, but our standing assumption is that such do not exist on $(M, g)$.

Using standard elliptic theory one can prove the following Proposition, we leave out the details of the argument.

Proposition 1.2. The set of metrics $g$ and functions $\tau$ for which Equation (6) has no solution is open with respect to the $C^{1}$-topology.

We conclude that Theorem 1.1 not only tells us that (2a)-(2b) have a solution if the limit equation has no solution, but also that $g, \tau, \sigma$ are in the interior of the set of data for which (2a)-(2b) have solutions. 
Note that we do not make any claim about uniqueness. Since our construction use the Schauder fixed point theorem as a central step we cannot draw any conclusion about the uniqueness of solutions.

We continue with some applications of Theorem 1.1

Corollary 1.3. Let data be given on $M$ as specified in (41) and assume that (5D) holds. Let $\lambda>0$. Assume that the Ricci curvature Ric of $(M, g)$ satisfies $\operatorname{Ric} \leq-\lambda g$ and that

$$
\left\|\frac{d \tau}{\tau}\right\|_{L^{\infty}}<\sqrt{\frac{n}{2(n-1)} \lambda}
$$

then the system of equations (2a) -(2b) admits a solution $(\varphi, W)$ with $\varphi>0$. Furthermore, the set of solutions $(\varphi, W) \in W_{+}^{2, p} \times W^{2, p}$ is compact.

The next result is less explicit but holds without any curvature assumptions. We define

$$
C_{g}:=\inf \frac{\left(\int_{M}|L V|^{2} d v\right)^{\frac{1}{2}}}{\left(\int_{M}|V|^{N} d v\right)^{\frac{1}{N}}},
$$

where the infimum is taken over all smooth 1 -forms $V$ on $M$ with $V \not \equiv 0$. By standard elliptic estimates the constant $C_{g}$ is positive if there are no conformal Killing vector fields on $(M, g)$, see Lemma A.1 in the Appendix.

Corollary 1.4. Let data be given on $M$ as specified in (4) and assume that (5) holds. If

$$
\left\|\frac{d \tau}{\tau}\right\|_{L^{n}}<\frac{1}{2} \sqrt{\frac{n}{n-1}} C_{g}
$$

then the system of equations (2a) $-(2 \mathrm{~b})$ admits a solution $(\varphi, W)$ with $\varphi>0$. Furthermore, the set of solutions $(\varphi, W) \in W_{+}^{2, p} \times W^{2, p}$ is compact.

Since we do not know all the solutions of (2a) $-(2 \mathrm{~b})$ a natural question is the following: given $M, \tau$, and $\sigma$ as above, what can we say about the set of metrics $g$ for which the system of equations (2a)-(2b) has a solution? Is it a large set in some appropriate sense? The next result gives a partial answer to this question.

Corollary 1.5. Let $\tau \in W^{1, p}$ be a function on $M$ which vanishes nowhere. Let $\mathcal{R}(M, \tau)$ be the set of metrics on $M$ such that for all symmetric, trace- and divergencefree $(0,2)$-tensors $\sigma \in W^{1, p}$ the assumptions (5) hold and the system of equations (2a) - (2b) admits a solution with $\varphi>0$. Then the set $\mathcal{R}(M, \tau)$ is dense with respect to the $C^{0}$-topology in the set $\mathcal{R}(M)$ of all metrics on $M$.

Corollary 1.5 leads to the question if the set $\mathcal{R}(M, \tau, \sigma)$ is dense in a stronger topology. From a physical point of view, the gravitational field depends on the LeviCivita connection and hence on the first derivatives of $g$. So, a $C^{1}$-density result would be interesting. At the moment, we are not able to obtain such a statement. One could speculate that the limit equation (6) never admits a non-trivial solution. The following proposition says that this is not true.

Proposition 1.6. On the sphere $S^{n}$ there exists a metric $g$ and a function $\tau$ such that the limit equation (6) for $\tau, g$ has a non-trivial solution for some $\alpha_{0} \in(0,1]$.

The proof is surprisingly complicated, it uses a contradiction argument which might be unexpected for such an existence result. 
Our last result is not related to the limit equation. It is a near-CMC nonexistence result which strengthens previous results of J. Isenberg and N. Ó Murchadha [15, Theorem 2].

Theorem 1.7. Let data be given on $M$ as specified in (4). Assume that $\tau$ vanishes nowhere, $(M, g)$ has no conformal Killing vector fields, and the operator

$$
\frac{4(n-1)}{n-2} \frac{N+1}{\left(\frac{N}{2}+1\right)^{2}} \Delta+R=\frac{3 n-2}{n-1} \Delta+R
$$

is non-negative. Then if $\sigma \equiv 0$ and

$$
\left\|\frac{d \tau}{\tau}\right\|_{L^{n}}<\frac{1}{2} \sqrt{\frac{n}{n-1}} C_{g},
$$

there is no solution $(\varphi, W) \in W_{+}^{2, p} \times W^{2, p}$ of (2a) $-(2 \mathrm{~b})$.

The assumption of non-negativity of this operator is weaker than $R \geq 0$ but stronger than $Y(g) \geq 0$. This last fact is natural since the conformal method is not conformally covariant. Also, the assumptions made for Theorem 1.1 and Theorem 1.7 are mutually exclusive.

In Section 2 we introduce the subcritical perturbation of the conformal constraint equations and prove Theorem 1.1. In Section 3 we give proofs of the corollaries and of Proposition 1.6. Section 4 is devoted to a proof of the non-existence result in Theorem 1.7. and finally in the Appendix we prove that the constant $C_{g}$ is positive.

The methods of this paper will be applied to the vacuum constraint equations on asymptotically hyperbolic manifolds in [9]. The asymptotically euclidean case will be treated in a forthcoming paper.

We thank Jim Isenberg for showing us the idea of using subcritical perturbations of the conformal constraint equations. Also we thank Piotr Chruściel for pointing out a mistake in an earlier version of this article. Further, we would like to thank Bernd Ammann, Lars Andersson, Erwann Delay, and Olivier Druet for helpful discussions.

\section{Proof of Theorem 1.1}

In all of this section we assume that data is given on $M$ as specified in (4) and we assume that (5) holds. We will prove that the system (2a)-(2b) then admits a solution $(\varphi, W)$ with $\varphi>0$ if the limit equation does not admit any non-trivial solution. This proof proceeds in several steps.

2.1. The subcritical system. Let $0<\epsilon<1$. We begin by introducing the subcritical system where the exponent of $\varphi$ in $(2 \mathrm{~b})$ is decreased by $\epsilon$,

$$
\begin{aligned}
\frac{4(n-1)}{n-2} \Delta \varphi+R \varphi & =-\frac{n-1}{n} \tau^{2} \varphi^{N-1}+|\sigma+L W|^{2} \varphi^{-N-1}, \\
-\frac{1}{2} L^{*} L W & =\frac{n-1}{n} \varphi^{N-\epsilon} d \tau .
\end{aligned}
$$

In the following Proposition we follow the method of Maxwell [19] to show existence of solutions of these subcritical equations.

Proposition 2.1. Let data be given on $M$ as specified in (41) and assume that (5) holds. Also, let $0<\epsilon<1$. Then there exists at least one solution $(\varphi, W)$ of the subcritical constraint equations (7a) $-(7 \mathrm{~b})$. 
The proof of this proposition relies on a modified version of the Schauder fixed point theorem, see for example [10, Theorem 11.1, p. 279]. Let $\varphi \in L^{\infty}, \varphi>0$. By [19, Proposition 5] there exists a unique $W \in W^{2, p}$ such that

$$
-\frac{1}{2} L^{*} L W=\frac{n-1}{n} \varphi^{N-\epsilon} d \tau,
$$

and by [19, Proposition 2] there is a unique $\psi \in W_{+}^{2, p}$ satisfying

$$
\frac{4(n-1)}{n-2} \Delta \psi+R \psi=-\frac{n-1}{n} \tau^{2} \psi^{N-1}+|\sigma+L W|^{2} \psi^{-N-1} .
$$

We define

$$
N_{\epsilon}(\varphi):=\psi
$$

and prove the following result.

Lemma 2.2. There exists a constant $a_{\epsilon}>0$ such that for all $b \leq a_{\epsilon}$, there is a constant $K_{b}$ depending only on $b, g, \tau$, and $\sigma$, but not on $\epsilon$ such that

$$
K_{b} \leq N_{\epsilon}(\varphi) \leq a_{\epsilon}
$$

for any $\varphi$ satisfying $0<\varphi \leq b$. In addition if $Y(g)<0, K_{b}$ does not depend on $b$.

All the arguments for this proof can be found in [19, Proposition 10], even though a slightly weaker conclusion is formulated there. To prove Proposition 2.1 above we only need the weaker version, later we will apply Lemma 2.2 as stated here.

Proof. Let $\varphi$ be a positive function and let $W$ satisfy the perturbed vector equation (8). Assume that $\theta_{-}$is a subsolution and $\theta_{+}$is a supersolution of Equation (9) with $\theta_{-} \leq \theta_{+}$. Then it is well known that that there is a solution $\theta$ of Equation (9) which satisfies $\theta_{-} \leq \theta \leq \theta_{+}$, see for example [13. Since solutions of (9) are unique for a given $W$ we conclude that $\theta=N_{\epsilon}(\varphi)$ and $\theta_{-} \leq N_{\epsilon}(\varphi) \leq \theta_{+}$. To prove Lemma 2.2. we show that $\theta_{+}=a_{\epsilon}$ is a supersolution if $a_{\epsilon}$ is a large real number and if $0<b \leq a_{\epsilon}$ is such that $\varphi \leq b$, then we can find a subsolution $\theta_{-}$whose minimum $K_{b}$ depends only on $b$.

By standard elliptic theory, there exists a constant $C_{0}$ depending only on $g$ such that any solution $W$ of Equation (8) satisfies

$$
\|L W\|_{L^{\infty}} \leq C_{0}\|d \tau\|_{L^{p}}\|\varphi\|_{L^{\infty}}^{N-\epsilon} .
$$

Let $a_{\epsilon}>0$ be large enough so that

$$
R a_{\epsilon}+\frac{n-1}{n} \tau^{2} a_{\epsilon}^{N-1}-2\|\sigma\|_{L^{\infty}}^{2} a_{\epsilon}^{-N-1}-2 C_{0}^{2}\|d \tau\|_{L^{p}}^{2} a_{\epsilon}^{N-1-2 \epsilon} \geq 0,
$$

such an $a_{\epsilon}$ can be found since we assume that $\tau$ vanishes nowhere and $\epsilon>0$. At this step we crucially use that the equations have been made subcritical, here is also the only place we use this fact. We set $\theta_{+}:=a_{\epsilon}$, this is then a supersolution of Equation (9). We now find a subsolution, for this let $b \leq a_{\epsilon}$ and assume that $\varphi \leq b$.

We first study the case when $Y(g) \geq 0$. Then after an appropriate conformal change $\bar{g}:=e^{2 u} g$ the scalar curvature $\bar{R}$ is non-negative. As in [19, Proposition 10] we consider the solution $\eta$ of

$$
\frac{4(n-1)}{n-2} \Delta \eta+\left(R+\frac{n-1}{n} \tau^{2}\right) \eta=|\sigma+L W|^{2} .
$$


Under the conformal change this equation is equivalent to the equation

$$
\frac{4(n-1)}{n-2} \bar{\Delta} \bar{\eta}+\left(\bar{R}+\frac{n-1}{n} e^{-2 u} \tau^{2}\right) \bar{\eta}=e^{-(n+2) u / 2}|\sigma+L W|^{2}
$$

for $\bar{\eta}:=e^{-(n-2) u / 2} \eta$. Since $\bar{R}+\frac{n-1}{n} e^{-2 u} \tau^{2}$ is positive we know there exists a positive solution $\bar{\eta}$ of this equation, and thus there exists a positive solution $\eta$ of Equation (11). We set $\eta_{\alpha}:=\alpha \eta$ for a positive constant $\alpha$, so that

$$
\begin{aligned}
& \frac{4(n-1)}{n-2} \Delta \eta_{\alpha}+R \eta_{\alpha}+\frac{n-1}{n} \tau^{2} \eta_{\alpha}^{N-1}-|\sigma+L W|^{2} \eta_{\alpha}^{-N-1} \\
& \quad=\frac{n-1}{n} \tau^{2}\left(\alpha^{N-1} \eta^{N-1}-\alpha \eta\right)+|\sigma+L W|^{2}\left(\alpha-\alpha^{-N-1} \eta^{-N-1}\right) .
\end{aligned}
$$

Choose $\alpha$ small enough so that this is non-positive, then $\theta_{-}:=\alpha \eta$ is a subsolution of Equation (9). The choice of the $\alpha$ depends only on $\max (\eta)$, so the constant $K_{b}:=\min \theta_{-}$depends only on $\max (\eta)$ and $\min (\eta)$. By [19, Proposition 9] we have

$$
\max (\eta) \leq C_{1}\left\||\sigma+L W|^{2}\right\|_{L^{p}} \leq C_{1}^{\prime}\left(\|\sigma\|_{L^{2 p}}^{\frac{1}{2}}+\|L W\|_{L^{2 p}}^{\frac{1}{2}}\right),
$$

and

$$
\min (\eta) \geq C_{2} \int_{M}|\sigma+L W|^{2} d v \geq C_{2} \int_{M}|\sigma|^{2} d v
$$

where $C_{1}, C_{2}$ depend only on $g$ and $\tau$. Using (10) we can further estimate

$$
\|L W\|_{L^{2 p}} \leq \operatorname{vol}(M)^{\frac{1}{2 p}}\|L W\|_{L^{\infty}} \leq \operatorname{vol}(M)^{\frac{1}{2 p}} C_{0}\|d \tau\|_{L^{p}}\|\varphi\|_{L^{\infty}}^{N-\epsilon} \leq C_{0}^{\prime}\|d \tau\|_{L^{p}} b^{N-\epsilon},
$$

where $C_{0}^{\prime}:=\operatorname{vol}(M)^{\frac{1}{2 p}} C_{0}$, and we conclude that $K_{b}$ only depends on $b, g, \tau$, and $\sigma$. This finishes the proof in the first case.

Now we study the case when $Y(g)<0$. By [2, Theorem 6.7, p. 197] there exists a positive function $\eta$ such that $\widetilde{g}:=\eta^{N-2} g$ has scalar curvature $\widetilde{R}=-\frac{n-1}{n} \tau^{2}$. The function $\eta$ solves the equation

$$
\frac{4(n-1)}{n-2} \Delta \eta+R \eta=-\frac{n-1}{n} \tau^{2} \eta^{N-1},
$$

and it follows that $\theta_{-}:=\eta$ is a subsolution of Equation (9). Note that in this case, $K_{b}=\min \theta_{-}$does not depend on $b$. This ends the proof of Lemma 2.2 .

The next step is to prove that the map $N_{\epsilon}$ is continuous. For this we define maps

$$
\Upsilon_{\epsilon}: L_{+}^{\infty} \ni \varphi \mapsto W \in W^{2, p}
$$

where $W$ is the unique solution of the perturbed vector equation (8) given $\varphi$, and

$$
\Lambda_{\epsilon}: C^{1} \ni W \mapsto \psi \in L_{+}^{\infty}
$$

where $\psi$ is the unique solution of the Lichnerowicz equation (9) given $W$. Note that $N_{\epsilon}=\Lambda_{\epsilon} \circ I_{p} \circ \Upsilon_{\epsilon}$ where $I_{p}: W^{2, p} \rightarrow C^{1}$ is the Sobolev injection.

Lemma 2.3. The maps $\Upsilon_{\epsilon}, \Lambda_{\epsilon}$, and thus also $N_{\epsilon}$, are continuous.

Proof. The map $\Upsilon_{\epsilon}: \varphi \mapsto W=\left(-\frac{1}{2} L^{*} L\right)^{-1}\left((n-1) \varphi^{N-\epsilon} d \tau\right)$ can be decomposed as

$$
\begin{aligned}
& L_{+}^{\infty} \quad \rightarrow \quad L_{+}^{\infty} \quad \rightarrow \quad L^{p} \quad \rightarrow \quad W^{2, p} \\
& \varphi \mapsto \varphi^{N-\epsilon} \mapsto(n-1) \varphi^{N-\epsilon} d \tau \quad \mapsto \quad\left(-\frac{1}{2} L^{*} L\right)^{-1}\left((n-1) \varphi^{N-\epsilon} d \tau\right)
\end{aligned}
$$

and the continuity of each arrow here is straightforward. 
Next we prove that $\Lambda_{\epsilon}$ is continuous, we give a proof which is independent of the Yamabe class of $g$. Let $W_{i} \in C^{1}, i=0,1$, be arbitrary. Denote the corresponding solutions of Equation (9) by $\psi_{i}$ and set $u_{i}=\ln \psi_{i}$. Then $u_{i}$ satisfies the equation

$$
\frac{4(n-1)}{n-2}\left(\Delta u_{i}+\left|d u_{i}\right|^{2}\right)+R=-\frac{n-1}{n} \tau^{2} e^{(N-2) u_{i}}+\left|\sigma+L W_{1}\right|^{2} e^{-(N+2) u_{i}} .
$$

Subtracting the equation for $u_{0}$ from the equation for $u_{1}$ we obtain

$$
\begin{aligned}
& \frac{4(n-1)}{n-2}\left(\Delta\left(u_{1}-u_{0}\right)+\left\langle d\left(u_{1}+u_{0}\right), d\left(u_{1}-u_{0}\right)\right\rangle\right) \\
& \quad=-\frac{n-1}{n} \tau^{2}\left(e^{(N-2) u_{1}}-e^{(N-2) u_{0}}\right) \\
& \quad+\left|\sigma+L W_{1}\right|^{2} e^{-(N+2) u_{1}}-\left|\sigma+L W_{2}\right|^{2} e^{-(N+2) u_{0}} .
\end{aligned}
$$

Setting $u_{\lambda}:=(1-\lambda) u_{0}+\lambda u_{1}$ for $0 \leq \lambda \leq 1$ the previous equation can be rewritten as

$$
\begin{aligned}
\left(\left|\sigma+L W_{1}\right|^{2}-\left|\sigma+L W_{0}\right|^{2}\right) e^{-(N+2) u_{0}} & \\
= & \frac{4(n-1)}{n-2}\left(\Delta\left(u_{1}-u_{0}\right)+\left\langle d\left(u_{1}+u_{0}\right), d\left(u_{1}-u_{0}\right)\right\rangle\right) \\
& \quad+\frac{1}{N-2} \frac{n-1}{n} \tau^{2}\left(u_{1}-u_{0}\right) \int_{0}^{1} e^{(N-2) u_{\lambda}} d \lambda \\
& \quad+\frac{1}{N+2}\left|\sigma+L W_{1}\right|^{2}\left(u_{1}-u_{0}\right) \int_{0}^{1} e^{-(N+2) u_{\lambda}} d \lambda .
\end{aligned}
$$

Note that by Lemma 2.2 we have $\psi_{i} \geq K_{b}$, so $e^{(N-2) u_{\lambda}} \geq K_{b}^{N-2}$, and

$$
\begin{gathered}
\frac{1}{N-2} \frac{n-1}{n} \tau^{2} \int_{0}^{1} e^{(N-2) u_{\lambda}} d \lambda+\frac{1}{N+2}\left|\sigma+L W_{1}\right|^{2} \int_{0}^{1} e^{-(N+2) u_{\lambda}} d \lambda \\
\geq c_{b}:=\frac{1}{N-2} \frac{n-1}{n} \tau_{0}^{2} K_{b}^{N-2},
\end{gathered}
$$

where $\tau_{0}^{2}:=\min _{M} \tau^{2}$ is positive by assumption. We are now in a position to apply the maximum principle [10, Theorem 8.1, p. 179] to the function $u_{1}-u_{0}$ and we obtain

$$
\begin{aligned}
\left\|u_{1}-u_{0}\right\|_{L^{\infty}} & \leq \frac{1}{c_{b}}\left\|\left(\left|\sigma+L W_{1}\right|^{2}-\left|\sigma+L W_{0}\right|^{2}\right) e^{-(N+2) u_{0}}\right\|_{L^{\infty}} \\
& \leq \frac{b^{-N-2}}{c_{b}}\left\|\left|\sigma+L W_{1}\right|^{2}-\left|\sigma+L W_{0}\right|^{2}\right\|_{L^{\infty}} \\
& \leq \frac{b^{-N-2}}{c_{b}}\left\|2 \sigma+L\left(W_{1}+W_{0}\right)\right\|_{L^{\infty}}\left\|L\left(W_{1}-W_{0}\right)\right\|_{L^{\infty}} \\
& \leq \frac{2 b^{-N-2}}{c_{b}}\left\|2 \sigma+L\left(W_{1}+W_{0}\right)\right\|_{L^{\infty}}\left\|W_{1}-W_{0}\right\|_{C^{1}} .
\end{aligned}
$$

This proves that the map $\Lambda_{\epsilon}: W \mapsto \psi$ is Lipschitz continuous on any subset $\left\{W \in C^{1} \mid\|W\|_{C^{1}} \leq C\right\}$ for any $C$ large enough.

We now prove the existence of solutions to the subcritical system.

Proof of Proposition [2.1, We follow the arguments of [19, Section 4.2] and define $U:=\left\{\varphi \in L^{\infty} \mid K_{a_{\epsilon}} \leq \varphi \leq a_{\epsilon}\right\}$. Here $K_{a_{\epsilon}}$ is the constant given by Lemma 2.2 applied with $b=a_{\epsilon}$. From Lemma 2.2 $N_{\epsilon}$ maps $U$ into itself. The set $\Upsilon_{\epsilon}(U)$ 
is bounded. Because of the Rellich-Kondrakov theorem, $I_{p} \circ \Upsilon_{\epsilon}(U)$ is relatively compact. Hence $N_{\epsilon}(U)=\Lambda_{\epsilon} \circ I_{p} \circ \Upsilon_{\epsilon}(U) \subset U$ is also relatively compact. From the fact that $U$ is a convex closed subset of $L^{\infty}$, the closed convex hull $V$ of $N_{\epsilon}(U)$ is contained in $U$, hence $V$ is a compact convex subset and the map $N_{\epsilon}$ maps $V$ into itself. By the Schauder fixed point theorem, there exists a fixed point for $N_{\epsilon}$ in $V$, namely a couple $\left(\varphi_{\epsilon}, W_{\epsilon}\right), \varphi_{\epsilon}>0$, solving the system (7a)-(7b). By standard regularity theorems we get $\varphi_{\epsilon} \in W_{+}^{2, p}$ and $W_{\epsilon} \in W^{2, p}$.

2.2. Convergence of subcritical solutions. Let $\epsilon \in[0,1)$ be arbitrary and let $(\varphi, W)$ be a solution of the subcritical equations (7a)-(7b). Define the energy of this solution by

$$
\gamma(\varphi, W):=\int_{M}|L W|^{2} d v
$$

and set $\widetilde{\gamma}:=\max \{\gamma, 1\}$. We first prove that $\widetilde{\gamma}$ controls the $L^{\infty}$-norm of $\varphi$. Note that $\epsilon=0$ is allowed, so the result applies also to solutions of (2a) $-(2 \mathrm{~b})$.

Proposition 2.4. There exists a constant $C$ such that for any $\epsilon \in[0,1)$ and any pair $(\varphi, W)$ solving the subcritical equations (7a) -(7b) it holds that

$$
\varphi \leq C \widetilde{\gamma}(\varphi, W)^{\frac{1}{2 N}} .
$$

Proof. To abbreviate we set $\widetilde{\gamma}:=\widetilde{\gamma}(\varphi, W)$. We rescale $\varphi, W$ and $\sigma$ as follows,

$$
\widetilde{\varphi}:=\widetilde{\gamma}^{-\frac{1}{2 N}} \varphi, \quad \widetilde{W}:=\widetilde{\gamma}^{-\frac{1}{2}} W, \quad \widetilde{\sigma}:=\widetilde{\gamma}^{-\frac{1}{2}} \sigma .
$$

The subcritical equations (7a) $-(7 \mathrm{~b})$ can then be rewritten as

$$
\begin{aligned}
\frac{1}{\widetilde{\gamma}^{\frac{1}{n}}}\left(\frac{4(n-1)}{n-2} \Delta \widetilde{\varphi}+R \widetilde{\varphi}\right)+\frac{n-1}{n} \tau^{2} \widetilde{\varphi}^{N-1} & =|\widetilde{\sigma}+L \widetilde{W}|^{2} \widetilde{\varphi}^{-N-1}, \\
-\frac{1}{2} L^{*} L \widetilde{W} & =\frac{n-1}{n} \widetilde{\gamma}^{-\frac{\epsilon}{2 N}} \widetilde{\varphi}^{N-\epsilon} d \tau .
\end{aligned}
$$

Due to the rescaling we have

$$
\int_{M}|L \widetilde{W}|^{2} d v \leq 1
$$

The proof proceeds in three steps.

Step 1. Bound for the $L^{2}$-norm of $\widetilde{\varphi}^{N}$

Multiplying equation (13a) by $\widetilde{\varphi}^{N+1}$ and integrating over $M$, we obtain

$$
\begin{aligned}
& \frac{1}{\widetilde{\gamma}^{\frac{1}{n}}} \int_{M}\left(\frac{4(n-1)}{n-2} \widetilde{\varphi}^{N+1} \Delta \widetilde{\varphi}+R \widetilde{\varphi}^{N+2}\right) d v+\frac{n-1}{n} \int_{M} \tau^{2} \widetilde{\varphi}^{2 N} d v \\
& \quad=\int_{M}|\widetilde{\sigma}+L \widetilde{W}|^{2} d v \\
& \quad=\int_{M}|\widetilde{\sigma}|^{2} d v+\int_{M}|L \widetilde{W}|^{2} d v \\
& \quad \leq \int_{M}|\widetilde{\sigma}|^{2} d v+1 .
\end{aligned}
$$

Integration by parts tells us that the first term in the first integral is non-negative. We set $\tau_{0}^{2}:=\min _{M} \tau^{2}$, which is positive by assumption, and conclude that

$$
\frac{1}{\widetilde{\gamma}^{\frac{1}{n}}} \int_{M} R \widetilde{\varphi}^{N+2} d v+\frac{n-1}{n} \tau_{0}^{2} \int_{M} \widetilde{\varphi}^{2 N} d v \leq \int_{M}|\widetilde{\sigma}|^{2} d v+1 .
$$


Here the first term can be estimated using the Hölder inequality

$$
\left|\int_{M} R \widetilde{\varphi}^{N+2} d v\right| \leq\left(\int_{M}|R|^{\frac{2 N}{N-2}} d v\right)^{\frac{N-2}{2 N}}\left(\int_{M} \widetilde{\varphi}^{2 N} d v\right)^{\frac{N+2}{2 N}}
$$

Remark that $\frac{2 N}{N-2}=n<p$, hence, setting

$$
\lambda=\left.\left.\left|\int_{M}\right| R\right|^{n}\right|^{\frac{1}{n}}<\infty
$$

we obtain:

$$
-\frac{\lambda}{\widetilde{\gamma}^{\frac{1}{n}}}\left(\int_{M} \widetilde{\varphi}^{2 N} d v\right)^{\frac{N+2}{2 N}}+\frac{n-1}{n} \tau_{0}^{2} \int_{M} \widetilde{\varphi}^{2 N} d v \leq \int_{M}|\widetilde{\sigma}|^{2} d v+1 .
$$

Since $\frac{N+2}{2 N}<1$ and $\widetilde{\gamma} \geq 1$ by definition, we obtain that the $L^{2}$-norm of $\widetilde{\varphi}^{N}$ is bounded independently of the choice of $\epsilon, \varphi$, and $W$.

\section{Step 2. Induction step}

We shall now show that the previous $L^{2}$-estimate for $\varphi^{N}$ can be iteratively improved to give $L^{p}$-estimates for $p \geq 2$. Set $p_{0}=2$, we construct inductively an increasing sequence $p_{i}$ such that $\widetilde{\varphi}^{N}$ is bounded in the $L^{p_{i}}$-norm. Assume by induction that $\widetilde{\varphi}^{N}$ is bounded in $L^{p_{i}}$. We first improve our estimate for $\widetilde{W}$. From Equation (13b) we get

$$
\frac{1}{2}\left\|L^{*} L \widetilde{W}\right\|_{L^{q_{i}}} \leq \frac{n-1}{n}\left\|\widetilde{\varphi}^{N-\epsilon}\right\|_{L^{p_{i}}}\|d \tau\|_{L^{p}},
$$

where $q_{i}$ is such that $\frac{1}{q_{i}}=\frac{1}{p_{i}}+\frac{1}{p}$. Note that the sequence $q_{i}$ is increasing since $p_{i}$ is and that $\frac{1}{q_{0}}=\frac{1}{2}+\frac{1}{p}<1$ since $p>n \geq 3$. From Young's inequality, we infer

$$
\widetilde{\varphi}^{N-\epsilon} \leq \frac{N-\epsilon}{N} \widetilde{\varphi}^{N}+\frac{\epsilon}{N}
$$

hence

$$
\left\|\widetilde{\varphi}^{N-\epsilon}\right\|_{p_{i}} \leq \frac{N-\epsilon}{N}\left\|\widetilde{\varphi}^{N}\right\|_{p_{i}}+\frac{\epsilon}{N} \operatorname{vol}(M)^{\frac{1}{p_{i}}} \leq\left\|\widetilde{\varphi}^{N}\right\|_{p_{i}}+\frac{1}{N} \max \{1, \operatorname{vol}(M)\} .
$$

From standard elliptic estimates and the fact that $(M, g)$ admits no conformal Killing vector field, there exists a constant $C_{i}$ such that

$$
\|\widetilde{W}\|_{W^{2, q_{i}}} \leq C_{i}\left(\left\|\widetilde{\varphi}^{N}\right\|_{p_{i}}+\frac{1}{N} \max \{1, \operatorname{vol}(M)\}\right)\|d \tau\|_{L^{p}} .
$$

Assume that $q_{i}<n$. By the Sobolev injection, we deduce that $L \widetilde{W}$ is bounded in $L^{r_{i}}$ where $r_{i}$ is such that $\frac{1}{r_{i}}=\frac{1}{q_{i}}-\frac{1}{n}$. We now estimate $\widetilde{\varphi}^{N}$ by means of the rescaled Lichnerowicz equation (13a). The method is similar to Step 1 We multiply (13a) by $\widetilde{\varphi}^{N+1+N k_{i}}$ where $k_{i}$ satisfies $\frac{2}{r_{i}}+\frac{k_{i}}{p_{i}}=1$ and integrate over $M$,

$$
\begin{aligned}
\int_{M} \mid \widetilde{\sigma} & +\left.L \widetilde{W}\right|^{2} \widetilde{\varphi}^{N k_{i}} d v \\
= & \frac{1}{\widetilde{\gamma}^{\frac{1}{n}}} \int_{M}\left(\frac{4(n-1)}{n-2} \widetilde{\varphi}^{N+1+N k_{i}} \Delta \widetilde{\varphi}+R \widetilde{\varphi}^{N+2+N k_{i}}\right) d v \\
& \quad+\frac{n-1}{n} \int_{M} \tau^{2} \widetilde{\varphi}^{2 N+N k_{i}} d v
\end{aligned}
$$


from which we find

$$
\begin{aligned}
2 \int_{M} & \left(|\widetilde{\sigma}|^{2}+|L \widetilde{W}|^{2}\right) \widetilde{\varphi}^{N k_{i}} d v \\
\geq & \frac{1}{\widetilde{\gamma}^{\frac{1}{n}}} \int_{M}\left(\frac{4(n-1)}{n-2} \frac{N+1+N k_{i}}{\left(\frac{N+N k_{i}}{2}+1\right)^{2}}\left|d \widetilde{\varphi}^{\frac{N+N k_{i}}{2}+1}\right|^{2}+R \widetilde{\varphi}^{N+2+N k_{i}}\right) d v \\
& \quad+\frac{n-1}{n} \tau_{0}^{2} \int_{M} \widetilde{\varphi}^{2 N+N k_{i}} d v \\
\geq & \frac{1}{\widetilde{\gamma}^{\frac{1}{n}}} \int_{M} R \widetilde{\varphi}^{N+2+N k_{i}} d v+\frac{n-1}{n} \tau_{0}^{2} \int_{M} \widetilde{\varphi}^{2 N+N k_{i}} d v
\end{aligned}
$$

By a method similar to the one we used in Step 1, we obtain that

$$
\frac{1}{\widetilde{\gamma}^{\frac{1}{n}}} \int_{M} R \widetilde{\varphi}^{N+2+N k_{i}} d v \geq-\frac{C_{i}}{\widetilde{\gamma}^{\frac{1}{n}}}\left(\int_{M} \widetilde{\varphi}^{\left(2+k_{i}\right) N} d v\right)^{\frac{2+\left(1+k_{i}\right) N}{\left(2+k_{i}\right) N}}
$$

for some constant $C_{i}$ that depends only on $g$ and $k_{i}$. We get the following inequality

$$
\begin{aligned}
& -\frac{C_{i}}{\widetilde{\gamma}^{\frac{1}{n}}}\left(\int_{M} \widetilde{\varphi}^{\left(2+k_{i}\right) N} d v\right)^{\frac{2+\left(1+k_{i}\right) N}{\left(2+k_{i}\right) N}}+\frac{n-1}{n} \tau_{0}^{2} \int_{M} \widetilde{\varphi}^{2 N+N k_{i}} d v \\
& \quad \leq 2 \int_{M}\left(|\widetilde{\sigma}|^{2}+|L \widetilde{W}|^{2}\right) \widetilde{\varphi}^{N k_{i}} d v .
\end{aligned}
$$

Here we have that $|\widetilde{\sigma}|^{2}+|\widetilde{W}|^{2}$ is bounded in $L^{\frac{r_{i}}{2}}$ and $\widetilde{\varphi}^{N k_{i}}$ is bounded in $L^{\frac{p_{i}}{k_{i}}}$, by the Young inequality we conclude that the right hand side of (14) is bounded. From the fact that $N>2$, we have

$$
\frac{2+\left(1+k_{i}\right) N}{\left(2+k_{i}\right) N}<1
$$

Hence by Inequality (14), we conclude that $\widetilde{\varphi}^{N}$ is bounded in $L^{p_{i+1}}$ where $p_{i+1}=$ $2+k_{i}$. A simple calculation yields

$$
\frac{p_{i+1}}{p_{i}}=1+2\left(\frac{1}{n}-\frac{1}{p}\right)>1
$$

Since

$$
\frac{1}{q_{i}}=\frac{1}{p_{i}}+\frac{1}{p}<\frac{1}{p_{i}}+\frac{1}{n}
$$

there exists an $i_{0}>0$ such that $q_{i_{0}} \geq n$ and $q_{i_{0}-1}<n$. When $q_{i}>n$, the Sobolev space $W^{1, q_{i}}$ embeds into some Hölder space so the strategy changes. This is the content of the next step.

Step 3. $L^{\infty}$ bound

Assume first that $q_{i_{0}}>n$. Since $\widetilde{W}$ is bounded in $W^{2, q_{i_{0}}}$ we know that $L \widetilde{W}$ is bounded in the $C^{\alpha}$-norm where $\alpha:=1-\frac{n}{q_{i_{0}}}$. From the fact that the Laplacian acting on functions only involves first order derivatives of the metric, it can be easily seen that the function $\widetilde{\varphi}$ is in $C^{2, \alpha}$. We can thus apply the classical maximum principle to the equation (13a). Let $x \in M$ be such that $\widetilde{\varphi}$ attains its maximum value at $x$. At such a point, the following inequality holds

$$
\frac{1}{\widetilde{\gamma}^{\frac{1}{n}}} R \widetilde{\varphi}+\frac{n-1}{n} \tau^{2} \widetilde{\varphi}^{N-1} \leq|\widetilde{\sigma}+L \widetilde{W}|^{2} \widetilde{\varphi}^{-N-1},
$$


or

$$
\frac{1}{\widetilde{\gamma}^{\frac{1}{n}}} R \widetilde{\varphi}^{N+2}+\frac{n-1}{n} \tau^{2} \widetilde{\varphi}^{2 N} \leq|\widetilde{\sigma}+L \widetilde{W}|^{2} .
$$

Hence $\widetilde{\varphi}$ is bounded. The case $q_{i_{0}}=n$ can be avoided by slightly decreasing the value of $p$.

We have proved that $\widetilde{\varphi}$ is bounded by some constant which depends only on $g$, $\tau$, and $\sigma$. Since $\varphi=\widetilde{\gamma}^{\frac{1}{2 N}} \widetilde{\varphi}$ this proves Proposition 2.4.

We can now study what happens when $\epsilon \rightarrow 0$. This is the content of the next two lemmas. We will see that the behavior is determined by the fact that the corresponding energies $\gamma$ are bounded or not.

Lemma 2.5. Assume that there exists sequences $\epsilon_{i}$ and $\left(\varphi_{i}, W_{i}\right)$ such that $\epsilon_{i} \geq 0$, $\epsilon_{i} \rightarrow 0$, and $\left(\varphi_{i}, W_{i}\right)$ is a solution of the subcritical equations (7a)-(7b) with $\epsilon=\epsilon_{i}$. Furthermore assume $\gamma\left(\varphi_{i}, W_{i}\right)$ is bounded. Then there exists a subsequence of the $\left(\varphi_{i}, W_{i}\right)$ which converges in the $W^{2, p}$-norm to a solution $\left(\varphi_{\infty}, W_{\infty}\right)$ of the conformal constraint equations (2a) - (2b).

Proof. From the previous proposition, we know that the functions $\varphi_{i}$ are uniformly bounded for the $L^{\infty}$-norm. From Equation (7b), the sequence $W_{i}$ is uniformly bounded in $W^{2, p}$. Since $p>n$ we conclude by the Rellich-Kondrakov theorem that the map $L: W^{2, p} \rightarrow L^{\infty}$ is compact. Up to extracting a subsequence, we can assume that the sequence $L W_{i}$ converges for the $L^{\infty}$-norm to some $L W_{\infty}$. Hence, from Lemma 2.3, the functions $\varphi_{i}$ converge in the $W^{2, p}$-norm (and therefore in the $L^{\infty}$-norm) to some $\varphi_{\infty}$. Equation (7b) finally implies that the sequence $W_{i}$ converges to $W_{\infty}$ in the $W^{2, p}$-norm.

Lemma 2.6. Assume that there exists sequences $\epsilon_{i}$ and $\left(\varphi_{i}, W_{i}\right)$ such that $\epsilon_{i} \geq 0$, $\epsilon_{i} \rightarrow 0$, and $\left(\varphi_{i}, W_{i}\right)$ is a solution of the subcritical equations (7a) - (7b) with $\epsilon=\epsilon_{i}$. Furthermore assume $\gamma\left(\varphi_{i}, W_{i}\right) \rightarrow \infty$. Then there exists a non-zero solution $W \in$ $W^{2, p}$ of the limit equation

$$
-\frac{1}{2} L^{*} L W=\alpha_{0} \sqrt{\frac{n-1}{n}}|L W| \frac{d \tau}{\tau} .
$$

for some $\alpha_{0} \in(0,1]$.

Note that, heuristically, when $\alpha_{0}=1$, this equation is obtained from the constraint equations (2a) - (2b) by discarding the terms $\frac{4(n-1)}{n-2} \Delta \varphi+R \varphi$ and $\sigma$ in the Lichnerowicz equation (2a). These are the terms that disappear in the limit $\widetilde{\gamma} \rightarrow \infty$, see Equation (13a). The constant $\alpha_{0}$ will appear as a defect of scale invariance when dealing with subcritical systems, that is when $\epsilon_{i} \rightarrow 0$ but $\epsilon_{i} \neq 0$. More precisely, $\alpha_{0}$ will be defined as $\alpha_{0}:=\lim \gamma\left(\varphi_{i}, W_{i}\right)^{-\frac{\epsilon_{i}}{2 N}}$.

Proof. Arguing as in the previous lemma, the 1-forms $\widetilde{W}_{i}$ are uniformly bounded in $W^{2, p}$. Without loss of generality we can assume that $\gamma\left(\varphi_{i}, W_{i}\right)>1$ for all $i$, so that

$$
\int_{M}\left|L \widetilde{W}_{i}\right|^{2} d v=1
$$

Up to extracting a subsequence, we can then assume that $\widetilde{W}_{i}$ converges in the $C^{1}$-norm to some $\widetilde{W}_{\infty}$. Note that

$$
\int_{M}\left|L \widetilde{W}_{\infty}\right|^{2} d v=1
$$


so $\widetilde{W}_{\infty} \not \equiv 0$. All we have to show now is that the functions $\widetilde{\varphi}_{i}$ converge in the $L^{\infty}$-norm to $\widetilde{\varphi}_{\infty} \in L^{\infty}$, where $\widetilde{\varphi}_{\infty}$ is given by

$$
\widetilde{\varphi}_{\infty}^{N}=\sqrt{\frac{n}{n-1}} \tau^{-1}\left|L \widetilde{W}_{\infty}\right|
$$

in other words, $\widetilde{\varphi}_{\infty}$ is such that

$$
\frac{n-1}{n} \tau^{2} \widetilde{\varphi}_{\infty}^{N-1}=\left|L \widetilde{W}_{\infty}\right| \widetilde{\varphi}_{\infty}^{-N-1}
$$

If such a statement is proven, passing to the limit in $(13 \mathrm{~b})$, we see that $\widetilde{W}_{\infty}$ is a solution of the limit equation with $\alpha_{0}:=\lim \gamma\left(\varphi_{i}, W_{i}\right)^{-\frac{\epsilon_{i}}{2 N}}$ which belongs to $[0,1]$ since $\gamma\left(\varphi_{i}, W_{i}\right)$ is assumed to go to infinity. By assumption $(M, g)$ has no conformal Killing fields, which excludes the possibility that $\alpha_{0}=0$. Choose $\epsilon>0$, we will show that there exists $i_{0}$ such that

$$
\left|\widetilde{\varphi}_{i}-\widetilde{\varphi}_{\infty}\right|<\epsilon
$$

for all $i \geq i_{0}$. For this, take two arbitrary $C^{2}$ functions $\widetilde{\varphi}_{ \pm}$such that

$$
\begin{gathered}
\widetilde{\varphi}_{\infty}-\epsilon \leq \widetilde{\varphi}_{-} \leq \widetilde{\varphi}_{\infty}-\frac{\epsilon}{2}, \\
\widetilde{\varphi}_{\infty}+\frac{\epsilon}{2} \leq \widetilde{\varphi}_{+} \leq \widetilde{\varphi}_{\infty}+\epsilon .
\end{gathered}
$$

We first show that $\tilde{\varphi}_{+}$is a supersolution of the rescaled Lichnerowicz equation (13a) if $i$ is large enough. By the maximum principle, we conclude that

$$
\widetilde{\varphi}_{i} \leq \widetilde{\varphi}_{+} \leq \widetilde{\varphi}_{\infty}+\epsilon .
$$

Note first that $\widetilde{\varphi}_{+} \geq \frac{\epsilon}{2}>0$. Multiplying the rescaled Lichnerowicz equation (13a) by $\widetilde{\varphi}_{+}^{N+1}$, we have to show that

$$
\frac{\widetilde{\varphi}_{+}^{N+1}}{\widetilde{\gamma}^{\frac{1}{n}}}\left(\frac{4(n-1)}{n-2} \Delta \widetilde{\varphi}_{+}+R \widetilde{\varphi}_{+}\right)+\frac{n-1}{n} \tau^{2} \widetilde{\varphi}_{+}^{2 N} \geq\left|\widetilde{\sigma}+L \widetilde{W}_{i}\right|^{2} .
$$

Since

$$
\widetilde{\varphi}_{+}^{2 N} \geq\left(\widetilde{\varphi}_{\infty}+\frac{\epsilon}{2}\right)^{2 N} \geq \widetilde{\varphi}_{\infty}^{2 N}+\left(\frac{\epsilon}{2}\right)^{2 N}
$$

the previous inequality will be satisfied provided that

$$
\frac{\widetilde{\varphi}_{+}^{N+1}}{\widetilde{\gamma}^{\frac{1}{n}}}\left(\frac{4(n-1)}{n-2} \Delta \widetilde{\varphi}_{+}+R \widetilde{\varphi}_{+}\right)+\left(\frac{\epsilon}{2}\right)^{2 N} \geq\left|\widetilde{\sigma}+L \widetilde{W}_{i}\right|^{2}-\left|L \widetilde{W}_{\infty}\right|^{2} .
$$

Now note that both the terms

$$
\frac{\widetilde{\varphi}_{+}^{N+1}}{\widetilde{\gamma}^{\frac{1}{n}}}\left(\frac{4(n-1)}{n-2} \Delta \widetilde{\varphi}_{+}+R \widetilde{\varphi}_{+}\right)
$$

and

$$
\left|\widetilde{\sigma}+L \widetilde{W}_{i}\right|^{2}-\left|L \widetilde{W}_{\infty}\right|^{2}
$$

tend uniformly to zero due to the facts that $\widetilde{\varphi}_{+} \in C^{2}, \widetilde{\gamma}_{i} \rightarrow \infty$ and $\widetilde{W}_{i} \rightarrow \widetilde{W}_{\infty}$. This proves that there exists $i_{0}$ such that for all $i \geq i_{0}, \widetilde{\varphi}_{+}$is a supersolution.

We now prove that $\widetilde{\varphi}_{-} \leq \widetilde{\varphi}_{i}$ for $i$ large enough. Note first that $\widetilde{\varphi}_{i} \geq \gamma^{-\frac{1}{2 N}} K_{b}$ so this inequality will be fulfilled if we show that $\tilde{\varphi}_{-}$is a subsolution of the Lichnerowicz equation everywhere it is positive since we can then apply the maximum 
principle on the set $\left\{x \in M \mid \widetilde{\varphi}_{-}(x) \geq \gamma^{-\frac{1}{2 N}} K_{b}\right\}$. The proof is then exactly the same as for $\widetilde{\varphi}_{+}$, except that we use the inequality

$$
\widetilde{\varphi}_{-}^{2 N} \leq\left(\widetilde{\varphi}_{\infty}-\frac{\epsilon}{2}\right)^{2 N} \leq \widetilde{\varphi}_{\infty}^{2 N}-\left(\frac{\epsilon}{2}\right)^{2 N}
$$

We conclude that $\widetilde{\varphi}_{i}$ converges to $\widetilde{\varphi}_{\infty}$, which ends the proof of Lemma 2.6

With the results obtained so far we can now prove Theorem 1.1 .

Proof of Theorem 1.1. Assume that the limit equation (6) admits no non-zero solution for any $\alpha_{0} \in(0,1]$. We first show that there exists a non-zero solution of the constraint equations. Indeed, for positive integers $i$ set $\epsilon_{i}:=1 / i$. From Lemma2.1. there exists a solution $\left(\varphi_{i}, W_{i}\right) \in W_{+}^{2, p} \times W^{2, p}$ of the subcritical constraint equations (7a)-(7b) where $\epsilon=\epsilon_{i}$. If the sequence $\gamma\left(\varphi_{i}, W_{i}\right)$ was unbounded, there would exist a non-zero solution of the limit equation (6) by Lemma 2.6. This contradicts the assumptions of the proposition. Hence, the sequence $\gamma\left(\varphi_{i}, W_{i}\right)$ is bounded and Lemma 2.5 asserts that there exists a solution $\left(\varphi_{\infty}, W_{\infty}\right) \in W_{+}^{2, p} \times W^{2, p}$ of the constraint equations.

To prove compactness, let $\left(\varphi_{i}, W_{i}\right)$ be an arbitrary sequence of solutions of the conformal constraint equations. Applying Lemma 2.6 with $\epsilon_{i}=0$ we obtain that the sequence $\gamma\left(\varphi_{i}, W_{i}\right)$ is bounded. Lemma 2.5 then asserts that a subsequence of $\left(\varphi_{i}, W_{i}\right)$ converges. This ends the proof of Theorem 1.1

Remark 2.7. We see from the proof of Lemma 2.6 that $\alpha_{0}=1$ if the $\epsilon_{i}$ are all equal to 0 . This implies in particular if the limit equation (6) has no non-trivial solution for $\alpha_{0}=1$, then the compactness in the statement of Theorem 1.1 holds.

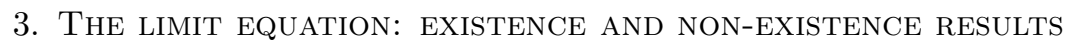

In this section, we study solutions of the limit equation (6). We first give nonexistence results and prove Corollaries 1.3, 1.4, and 1.5. Then, we derive some properties leading to the existence result in Proposition 1.6.

3.1. Non-existence results. We first prove the Limit equation has no solutions when the Ricci curvature has a negative upper bound.

Proof of Corollary 1.3. Under the assumption of Corollary 1.3. we show that the limit equation (6) does not have any non-trivial solution, Theorem 1.1 then implies the existence of solutions to the conformal constraint equations. We proceed by contradiction and assume that $W \not \equiv 0$ is a solution of

$$
-\frac{1}{2} L^{*} L W=\alpha_{0} \sqrt{\frac{n-1}{n}}|L W| \frac{d \tau}{\tau} .
$$

for some $\alpha_{0} \in(0,1]$. Taking the scalar product with $W$, integrating over $M$, and using the Hölder inequality, we get

$$
\frac{1}{2} \int_{M}|L W|^{2} d v \leq \sqrt{\frac{n-1}{n}}\left\|\frac{d \tau}{\tau}\right\|_{L^{\infty}}\left(\int_{M}|L W|^{2} d v\right)^{\frac{1}{2}}\left(\int_{M}|W|^{2} d v\right)^{\frac{1}{2}} .
$$

The conformal Killing operator satisfies the Bochner type formula

$$
\frac{1}{2} \int_{M}|L V|^{2} d v=\int_{M}\left(|\nabla V|^{2}+\left(1-\frac{2}{n}\right)|\operatorname{div} V|^{2}-\operatorname{Ric}(V, V)\right) d v,
$$


see for example [20, Chapter 2, §6]. Since we assume that the Ricci curvature satisfies Ric $\leq-\lambda g$ for some $\lambda>0$, we get

$$
\int_{M}|L W|^{2} d v \geq 2 \lambda \int_{M}|W|^{2} d v
$$

Together with (15), we obtain

$$
\left\|\frac{d \tau}{\tau}\right\|_{L^{\infty}} \geq \sqrt{\frac{n}{2(n-1)} \lambda}
$$

which contradicts the assumption of Corollary 1.3 .

The next result is that the limit equation has no solution under a near CMC condition.

Proof of Corollary 1.4 As in the proof of Corollary 1.3, we assume that $W$ is a solution of Equation (6). Taking the scalar product of this equation with $W$, integrating over $M$, and using the Hölder inequality, we find

$$
\frac{1}{2} \int_{M}|L W|^{2} d v \leq \sqrt{\frac{n-1}{n}}\left\|\frac{d \tau}{\tau}\right\|_{L^{n}}\left(\int_{M}|L W|^{2} d v\right)^{\frac{1}{2}}\left(\int_{M}|W|^{N} d v\right)^{\frac{1}{N}} .
$$

The definition of $C_{g}$ immediately implies that

$$
\left\|\frac{d \tau}{\tau}\right\|_{L^{n}} \geq \frac{1}{2} \sqrt{\frac{n}{n-1}} C_{g}
$$

which contradicts the assumption of Corollary 1.4.

Finally we show that metrics with no solution to the limit equation are dense in the $C^{0}$-topology.

Proof of Corollary 1.5. Fix any metric $g$ and set

$$
c:=\left\|\frac{d \tau}{\tau}\right\|_{L^{\infty}(M, g)}^{2} .
$$

By a result of J. Lohkamp [17, Theorem B] the set of metrics with a given upper bound on the Ricci curvature is dense in the space of all metrics with respect to the $C^{0}$-topology. Thus we can take a sequence of metrics $g_{i}$ tending to $g$ in $C^{0}$ with the property that

$$
\operatorname{Ric}^{g_{i}} \leq-\lambda g_{i}
$$

for all $i$, where $\lambda:=\frac{4(n-1)}{n} c$. For $i$ large enough we have

$$
\left\|\frac{d \tau}{\tau}\right\|_{L^{\infty}\left(M, g_{i}\right)}^{2} \leq \frac{3}{2} c<2 c=\frac{n}{2(n-1)} \lambda .
$$

By Corollary 1.3 we obtain the limit equation (6) has no non-trivial solutions for all metrics $g_{i}$ with $i$ large enough and hence, that the system of equations (2a)-(2b) admits a solution for all metrics $g_{i}$ with $i$ large enough. This proves Corollary 1.5 . 
3.2. Existence result for the limit equation. For the proof of Proposition 1.6 we will use the following result.

Proposition 3.1. Let $M$ be a compact n-manifold equipped with a smooth Riemannian metric $g$. Let also $\tau>0$ be smooth. Then at least one of the following statements is true.

- The limit equation (6) has a non-trivial solution for some $\alpha_{0} \in(0,1]$.

- For every trace-free and divergence-free smooth $(0,2)$-tensor $\sigma \not \equiv 0$ there exists a solution $W \in W^{2, p}$ of the equation

$$
-\frac{1}{2} L^{*} L W=\sqrt{\frac{n-1}{n}}|\sigma+L W| \frac{d \tau}{\tau} .
$$

The assumptions on the regularity of $g, \tau, \sigma$ in Proposition 3.1 could be weakened. To simplify the proof we choose to assume smoothness of the data, which is enough for the example we want to find.

Proof. Assume that the limit equation (6) has no solution and take a trace-free and divergence-free $(0,2)$-tensor $\sigma \not \equiv 0$. We need to prove that (17) then has a solution. Theorem 1.1 implies that for all $a>0$ the conformal constraint equations for $(g, \tau, a \sigma)$ admit a solution. So, there exists $\left(\varphi_{a}, W_{a}\right)$ with $\varphi_{a}>0$ such that

$$
\begin{aligned}
\frac{4(n-1)}{n-2} \Delta \varphi_{a}+R \varphi_{a} & =-\frac{n-1}{n} \tau^{2} \varphi_{a}^{N-1}+\left|a \sigma+L W_{a}\right|^{2} \varphi_{a}^{-N-1}, \\
-\frac{1}{2} L^{*} L W_{a} & =\frac{n-1}{n} \varphi_{a}^{N} d \tau .
\end{aligned}
$$

We will obtain a solution of (17) by letting $a$ tend to $+\infty$. We set $\gamma_{a}:=a^{2}$ and rescale $\varphi_{a}$ and $W_{a}$ as

$$
\widetilde{\varphi}_{a}:=\gamma_{a}^{-\frac{1}{2 N}} \varphi_{a}, \quad \widetilde{W}_{a}:=\gamma_{a}^{-\frac{1}{2}} W_{a} .
$$

Equations (18a)-118b) can be rewritten as

$$
\begin{aligned}
\frac{1}{\gamma_{a}^{\frac{1}{n}}}\left(\frac{4(n-1)}{n-2} \Delta \widetilde{\varphi}_{a}+R \widetilde{\varphi}_{a}\right) & =-\frac{n-1}{n} \tau^{2} \widetilde{\varphi}_{a}^{N-1}+\left|\sigma+L \widetilde{W}_{a}\right|^{2} \widetilde{\varphi}_{a}^{-N-1}, \\
-\frac{1}{2} L^{*} L \widetilde{W}_{a} & =\frac{n-1}{n} \widetilde{\varphi}_{a}^{N} d \tau .
\end{aligned}
$$

We divide the argument in two cases.

Case 1. Assume that

$$
\limsup _{a \rightarrow \infty} \int_{M}\left|L \widetilde{W}_{a}\right|^{2} d v=+\infty
$$

This situation is very similar to the situation in the proof of Theorem 1.1 when the energy $\gamma(\varphi, W)$ is unbounded. Setting

$$
\gamma_{a}^{\prime}:=\max \left\{\int_{M}\left|L \widetilde{W}_{a}\right|^{2} d v, 1\right\}
$$

and defining $\widetilde{W}_{a}^{\prime}:=\left(\gamma_{a}^{\prime}\right)^{-\frac{1}{2}} \widetilde{W}_{a}$, one sees that $\widetilde{W}_{a}^{\prime}$ converges to a non-trivial solution of the limit equation ([6). Since this equation is assumed to have no solution except 0 , the first case cannot occur. 
Case 2. Assume that

$$
\limsup _{a \rightarrow \infty} \int_{M}\left|L \widetilde{W}_{a}\right|^{2} d v<+\infty .
$$

Since $\gamma_{a}$ tends to $+\infty$, the situation is again similar to the situation in the proof of Theorem 1.1 when the energy $\gamma(\varphi, W)$ is unbounded. The difference here is that the $\sigma$-term does not vanish in the limit. So, mimicking the proof of Theorem 1.1. one sees that $\widetilde{W}_{a}$ tends to a solution of Equation (17). Note that in Equation (17) should appear some $\alpha_{0} \in(0,1]$, but Remark 2.7 implies that $\alpha_{0}=1$.

We can now give an example showing that non-existence of solutions of the limit equation is a property which is not open in the $C^{2}$-topology.

Proof of Proposition 1.6. On $S^{n}=\left\{\left(x_{1}, \ldots, x_{n+1}\right) \in \mathbb{R}^{n+1} \mid \sum_{i=1}^{n+1} x_{1}^{2}=1\right\}$, we choose a fixed open neighborhood $V$ of the north pole $N:=(1,0, \ldots, 0)$ and the south pole $S:=(-1,0, \ldots, 0)$. We choose a metric $g$ which has no local conformal Killing field on $M \backslash V$ and coincides with the standard round metric in a neighborhood of the points $N$ and $S$. The results in [4 provide the existence of such a metric. Set $\tau:=e^{x_{1}}$. Note that $\tau>0$ and that the gradient of $\ln (\tau)$ is a conformal Killing field of $S^{n}$ equipped with its standard round metric. Further, the critical points of $\tau$ are $N$ and $S$. By construction of the metric $g$ we have $L(d \ln (\tau))=L(d \tau / \tau)=0$ on a neighborhood of $N$ and $S$. This implies that there exists a constant $C>0$ such that for all $x \in S^{n}$

$$
\left|L\left(\frac{d \tau}{\tau}\right)\right| \leq C\left|\frac{d \tau}{\tau}\right|^{2}
$$

everywhere on $S^{n}$.

We will show that $g$ together with $\tau^{a}$ for $a>0$ large enough satisfy the conclusion of Proposition 1.6. To do this we proceed by contradiction and assume that for every $a>0$, the limit equation (6) has no non-trivial solution for any $\alpha_{0} \in(0,1]$. By Proposition 3.1, this means that for all $a>0$ and for all trace-free and divergencefree $(0,2)$-tensor $\sigma_{a}$ there exists a non-trivial solution $W_{a} \in W^{2, p}$ of

$$
-\frac{1}{2} L^{*} L W_{a}=\sqrt{\frac{n-1}{n}}\left|\sigma_{a}+L W_{a}\right| \frac{d \tau^{a}}{\tau^{a}}=\sqrt{\frac{n-1}{n}} a\left|\sigma_{a}+L W_{a}\right| \frac{d \tau}{\tau}
$$

Now choose a fixed non-zero trace-free and divergence-free (0,2)-tensors $\sigma$ supported in $M \backslash V$. For the existence of $\sigma$ with such properties, see for example [8]. We choose $\sigma_{a}=a^{-1} \sigma$ and get from Equation (21) a non-trivial solution of

$$
-\frac{1}{2} L^{*} L W_{a}=\sqrt{\frac{n-1}{n}}\left|\sigma+a L W_{a}\right| \frac{d \tau}{\tau} .
$$

Take the scalar product of this equation with $d \tau / \tau$ and integrate. This gives

$$
\sqrt{\frac{n-1}{n}} \int_{S^{n}}\left|\sigma+a L W_{a}\right|\left|\frac{d \tau}{\tau}\right|_{g}^{2} d v^{g}=-\frac{1}{2} \int_{S^{n}}\left\langle L W_{a}, L(d \tau / \tau)\right\rangle d v^{g} .
$$

Together with Inequality (20), we obtain

$$
\int_{S^{n}}\left|\sigma+a L W_{a}\right|\left|\frac{d \tau}{\tau}\right|^{2} d v^{g} \leq C \int_{S^{n}}\left|\frac{d \tau}{\tau}\right|^{2}\left|L W_{a}\right| d v^{g},
$$


with maybe a new value of $C$. Since $\left|\sigma+a L W_{a}\right| \geq a\left|L W_{a}\right|-|\sigma|$ we conclude from Inequality (22) that

$$
(a-C) \int_{S^{n}}\left|\frac{d \tau}{\tau}\right|_{g}^{2}\left|L W_{a}\right| d v^{g} \leq \int_{S^{n}}|\sigma|\left|\frac{d \tau}{\tau}\right|_{g}^{2} d v^{g} .
$$

The right hand side here is bounded which shows that

$$
\lim _{a \rightarrow+\infty} \int_{S^{n}}\left|\frac{d \tau}{\tau}\right|_{g}^{2}\left|L W_{a}\right| d v^{g}=0,
$$

Inequality (22) then tells us that

$$
\lim _{a \rightarrow \infty} \int_{S^{n}}\left|\sigma+a L W_{a}\right|\left|\frac{d \tau}{\tau}\right|_{g}^{2} d v^{g}=0 .
$$

Since the critical set of $\tau$ is exactly $N$ and $S$ we have

$$
\left|\frac{d \tau}{\tau}\right|_{g}^{2} \geq \epsilon>0
$$

on $S^{n} \backslash V$. From (23) we obtain

$$
\lim _{a \rightarrow \infty} \int_{S^{n} \backslash V}\left|\sigma+a L W_{a}\right| d v^{g}=0 .
$$

Since $\sigma$ is supported in $S^{n} \backslash V$, we have

$$
\left|\int_{S^{n}}\left\langle\sigma, \sigma+a L W_{a}\right\rangle d v^{g}\right| \leq\|\sigma\|_{L^{\infty}} \int_{S^{n} \backslash V}\left|\sigma+a L W_{a}\right| d v^{g} .
$$

Together with (24), this shows that

$$
\liminf _{a \rightarrow \infty} \int_{S^{n}}\left\langle\sigma, a L W_{a}\right\rangle d v^{g}=-\liminf _{a \rightarrow \infty} \int_{S^{n}}|\sigma|^{2} d v^{g} .
$$

We get that

$$
\liminf _{a \rightarrow \infty} \int_{S^{n}}\left\langle\sigma, a L W_{a}\right\rangle d v^{g} \neq 0 .
$$

However, since $\sigma$ is divergence-free, we must have

$$
\int_{S^{n}}\left\langle\sigma, a L W_{a}\right\rangle d v^{g}=0
$$

for all $a$, which gives the desired contradiction.

\section{Proof of Theorem 1.7}

Proof of Theorem 1.7. We proceed by contradiction and assume that the system (2a) -(2b) admits a solution $(\varphi, W)$ where $\varphi>0$. Note that

$$
\int_{M} \varphi^{N+1} \Delta \varphi d v=\frac{N+1}{\left(\frac{N}{2}+1\right)^{2}} \int_{M}\left|d \varphi^{\frac{N}{2}+1}\right|^{2} d v .
$$


Hence, multiplying the Lichnerowicz equation (2a) by $\varphi^{N+1}$ and integrating over $M$ we get

$$
\begin{aligned}
& \frac{4(n-1)}{n-2} \frac{N+1}{\left(\frac{N}{2}+1\right)^{2}} \int_{M}\left|d \varphi^{\frac{N}{2}+1}\right|^{2} d v+\int_{M} R \varphi^{N+2} d v+\frac{n-1}{n} \int_{M} \tau^{2} \varphi^{2 N} d v \\
& =\int_{M}|L W|^{2} d v
\end{aligned}
$$

since $\sigma \equiv 0$. By assumption the sum of the first two terms is non-negative, and we obtain

$$
\frac{n-1}{n} \int_{M} \tau^{2} \varphi^{2 N} d v \leq \int_{M}|L W|^{2} d v
$$

Next, we take the scalar product of the vector equation (2b) with $W$ and integrate over $M$. Using the Hölder inequality we get

$$
\begin{aligned}
\frac{1}{2} \int_{M}|L W|^{2} d v & =\frac{n-1}{n} \int_{M} \varphi^{N}\langle d \tau, W\rangle d v \\
& \leq \frac{n-1}{n}\left(\int_{M} \tau^{2} \varphi^{2 N} d v\right)^{1 / 2}\left(\int_{M}\left|\frac{d \tau}{\tau}\right|^{n} d v\right)^{\frac{1}{n}}\left(\int_{M}|W|^{N} d v\right)^{\frac{1}{N}}
\end{aligned}
$$

Using (25) and the definition of $C_{g}$ we obtain

$$
\frac{1}{2} C_{g} \leq \frac{1}{2} \frac{\left(\int_{M}|L W|^{2} d v\right)^{\frac{1}{2}}}{\left(\int_{M}|W|^{N} d v\right)^{\frac{1}{N}}} \leq \sqrt{\frac{n-1}{n}}\left\|\frac{d \tau}{\tau}\right\|_{L^{n}},
$$

since $W \not \equiv 0$. This contradicts the assumption and proves Theorem 1.7

\section{Appendix A. Positivity of $C_{g}$}

Lemma A.1. Suppose that $(M, g)$ has no conformal Killing vector fields, then $C_{g}>0$.

Proof. Since $M$ is compact there is a constant $\lambda$ such that Ric $\leq \lambda g$. From Formula (16) we get

$$
\frac{1}{2} \int_{M}|L V|^{2} d v \geq \int_{M}|\nabla V|^{2} d v-\lambda \int_{M}|V|^{2} d v
$$

and

$$
\frac{1}{2} \int_{M}|L V|^{2} d v \geq\|V\|_{H^{1}}^{2}-(\lambda+1)\|V\|_{L^{2}}^{2} .
$$

We argue by contradiction and assume that for all positive integers $k$ there exists $V_{k} \in H^{1}$ such that $\left\|V_{k}\right\|_{L^{N}}=1$ and $\frac{1}{2} \int_{M}\left|L V_{k}\right|^{2} d v \leq 1 / k$. Since $M$ is compact it follows that $V_{k}$ is uniformly bounded in $L^{2}$, and from Equation (26) we conclude that the sequence $V_{k}$ is uniformly bounded in $H^{1}$. By the compactness of the embedding $H^{1} \rightarrow L^{2}$, we can assume that the sequence $V_{k}$ converges to some $V_{\infty}$ in the $L^{2}$-norm. By continuity of the norm $V_{\infty}$ satisfies $\left\|V_{\infty}\right\|_{L^{2}}=1$, and in 
particular, $V_{\infty} \not \equiv 0$. For any smooth 1 -form $\xi$ we have

$$
\begin{aligned}
\frac{1}{2}\left|\int_{M}\left\langle L^{*} L \xi, V_{\infty}\right\rangle d v\right| & =\frac{1}{2} \lim _{k \rightarrow \infty}\left|\int_{M}\left\langle L^{*} L \xi, V_{k}\right\rangle d v\right| \\
& =\frac{1}{2} \lim _{k \rightarrow \infty}\left|\int_{M}\left\langle L \xi, L V_{k}\right\rangle d v\right| \\
& \leq \lim _{k \rightarrow \infty}\left(\frac{1}{2} \int_{M}|L \xi|^{2} d v\right)^{\frac{1}{2}}\left(\frac{1}{2} \int_{M}\left|L V_{k}\right|^{2} d v\right)^{\frac{1}{2}} \\
& =0 .
\end{aligned}
$$

Hence $V_{\infty}$ satisfies the equation $L^{*} L V_{\infty}=0$ in the sense of distributions, so is a nonzero conformal Killing vector field. This is a contradiction which proves that $C_{g}>0$.

\section{REFERENCES}

[1] P. T. Allen, A. Clausen, and J. Isenberg, Near-constant mean curvature solutions of the Einstein constraint equations with non-negative Yamabe metrics, Classical Quantum Gravity 25 (2008), no. 7, 075009, 15.

[2] T. Aubin, Some nonlinear problems in Riemannian geometry, Springer Monographs in Mathematics, Springer-Verlag, Berlin, 1998.

[3] R. Bartnik and J. Isenberg, The constraint equations, The Einstein equations and the large scale behavior of gravitational fields, Birkhäuser, Basel, 2004, pp. 1-38.

[4] R. Beig, P. T. Chruściel, and R. Schoen, KIDs are non-generic, Ann. Henri Poincaré 6 (2005), no. $1,155-194$.

[5] Y. Choquet-Bruhat, General relativity and the Einstein equations, Oxford Mathematical Monographs, Oxford University Press, Oxford, 2009.

[6] Y. Choquet-Bruhat and R. Geroch, Global aspects of the Cauchy problem in general relativity, Comm. Math. Phys. 14 (1969), 329-335.

[7] Y. Choquet-Bruhat and J. W. York, Jr., The Cauchy problem, General relativity and gravitation, Vol. 1, Plenum, New York, 1980, pp. 99-172.

[8] E. Delay, Smooth compactly supported solutions of some underdetermined elliptic PDE, with gluing applications, http://arxiv.org/abs/1003.0535

[9] R. Gicquaud and A. Sakovich, A large class of non constant mean curvature solutions of the einstein constraint equations on an asymptotically hyperbolic manifold, http://arxiv.org/abs/1012.2246

[10] D. Gilbarg and N. S. Trudinger, Elliptic partial differential equations of second order, Classics in Mathematics, Springer-Verlag, Berlin, 2001, Reprint of the 1998 edition.

[11] M. J. Holst, G. Nagy, and G. Tsogtgerel, Far-from-constant mean curvature solutions of Einstein's constraint equations with positive Yamabe metrics, Phys. Rev. Lett. 100 (2008), no. 16, 161101, 4.

[12] _ Rough solutions of the Einstein constraints on closed manifolds without near-CMC conditions, Comm. Math. Phys. 288 (2009), no. 2, 547-613.

[13] J. Isenberg, Constant mean curvature solutions of the Einstein constraint equations on closed manifolds, Classical Quantum Gravity 12 (1995), no. 9, 2249-2274.

[14] J. Isenberg and V. Moncrief, Some results on nonconstant mean curvature solutions of the Einstein constraint equations, Physics on manifolds (Paris, 1992), Math. Phys. Stud., vol. 15, Kluwer Acad. Publ., Dordrecht, 1994, pp. 295-302.

[15] J. Isenberg and N. Ó Murchadha, Non-CMC conformal data sets which do not produce solutions of the Einstein constraint equations, Classical Quantum Gravity 21 (2004), no. 3, S233-S241, A spacetime safari: essays in honour of Vincent Moncrief.

[16] A. Lichnerowicz, L'intégration des équations de la gravitation relativiste et le problème des $n$ corps, J. Math. Pures Appl. (9) 23 (1944), 37-63.

[17] J. Lohkamp, Curvature h-principles, Ann. of Math. 142 (1995), no. 3, 457-498.

[18] D. Maxwell, Rough solutions of the Einstein constraint equations, J. Reine Angew. Math. 590 (2006), 1-29. 
[19] _ A class of solutions of the vacuum Einstein constraint equations with freely specified mean curvature, Math. Res. Lett. 16 (2009), no. 4, 627-645.

[20] K. Yano, Integral formulas in Riemannian geometry, Pure and Applied Mathematics, No. 1, Marcel Dekker Inc., New York, 1970.

Institutionen för Matematik, Kungliga Tekniska Högskolan, 10044 Stockholm, SweDEN

E-mail address: dahl@math.kth.se

Laboratoire de Mathématiques et de Physique Théorique, UFR Sciences et Technologie, Faculté François Rabelais, Parc de Grandmont, 37200 Tours, France

E-mail address: romain.gicquaud@lmpt.univ-tours.fr

Institut Élie Cartan, BP 239, Université de NANCy 1, 54506 VAndoeuvre-LÈs-NanCy CEDEx, France

E-mail address: humbert@iecn.u-nancy.fr 